\title{
Denoising Large In Situ TEM Image Datasets: A Convolutional Neural Network- based Approach
}

\author{
Joshua Vincent $^{1}$, Sreyas Mohan ${ }^{2}$, Carlos Fernandez-Granda ${ }^{2}$ and Peter Crozier ${ }^{1}$ \\ ${ }^{1}$ Arizona State University, Tempe, Arizona, United States, ${ }^{2}$ New York University, New York City, New \\ York, United States
}

Heterogenous catalysts are an important class of materials that receive considerable research attention due to their large impact on energy and the environment. Aberration-corrected in situ environmental transmission electron microscopy (ETEM) is a powerful catalyst characterization tool capable of providing atomic-scale structural information from active catalysts under reaction conditions. Recent advancements in the realization of highly efficient direct electron detectors now enable atomicallyresolved ETEM images to be acquired with a temporal resolution in the millisecond or sub-millisecond regime, which is where catalytically relevant structural reconfigurations are thought to occur [1,2,3]. While there is potentially much to be gained from applying these new detectors to catalytic nanomaterials characterization, acquiring ETEM image series with high temporal resolution necessarily produces datasets that can be unwieldly in size and severely degraded by noise, rendering traditional image processing approaches impractical and ineffective at extracting useful scientific information. For example, recording a 64 megapixel in situ ETEM movie at 2.5 millisecond time resolution would produce around 100 gigabytes and many hundreds of frames of noisy image data every second. We are interested in exploring innovative approaches to image processing that leverage new advances in data science on large, noisy, atomic-resolution in situ ETEM data sets.

As a first step to processing in situ movies of catalyst dynamics, we have been developing a convolutional neural network to denoise large TEM image datasets. The network has been applied to a $\mathrm{CeO}_{2}$-supported Pt nanoparticle imaged in $\mathrm{N}_{2}$ gas with 25 millisecond temporal resolution. A training dataset was produced by generating 460 simulated HRTEM images of a Pt nanoparticle, which were then corrupted with a categorical noise model taken from the real images. Simulated images were generated with Dr. Probe [4]. Figure 1a shows an example of a simulated image which serves as a clean reference. The image corrupted with noise is shown in Figure 1b; this image is the input to the network. The denoised image predicted by the network is shown in Figure 1c. The network was trained to minimize the sum of the squared differences (i.e., L2 loss) between the clean reference and the predicted image; we used a network with 20 convolutional layers, each with 64 channels [5]. Compared to the training image, the denoised image has a peak signal-to-noise ratio of roughly 45 and a structural similarity index of 0.999 when averaged over 20 test images similar to the training data set.

The trained network was then applied to the real in situ TEM image dataset of the $\mathrm{CeO}_{2}$-supported Pt nanoparticle in $\mathrm{N}_{2}$ gas. Figure 2a and Figure 2b show a real 25 millisecond in situ TEM image and a denoised counterpart, respectively. Atomic columns corresponding to $\mathrm{Pt}$ are seen at the top half of the image, while columns corresponding to $\mathrm{CeO}_{2}$ are seen at the bottom. A 10 pixel integrated line profile was calculated across the sub-surface layer of Pt atomic columns in both the real and denoised image. The line profiles, displayed in Figure 2c, show that the denoised image displays a higher SNR than the real image and retains similar features. While still preliminary, the network-based approach may be immensely useful in denoising rapidly acquired in situ TEM images which suffer from a low signal-to-noise (e.g., those taken at $>1000$ frames per second) [6]. 

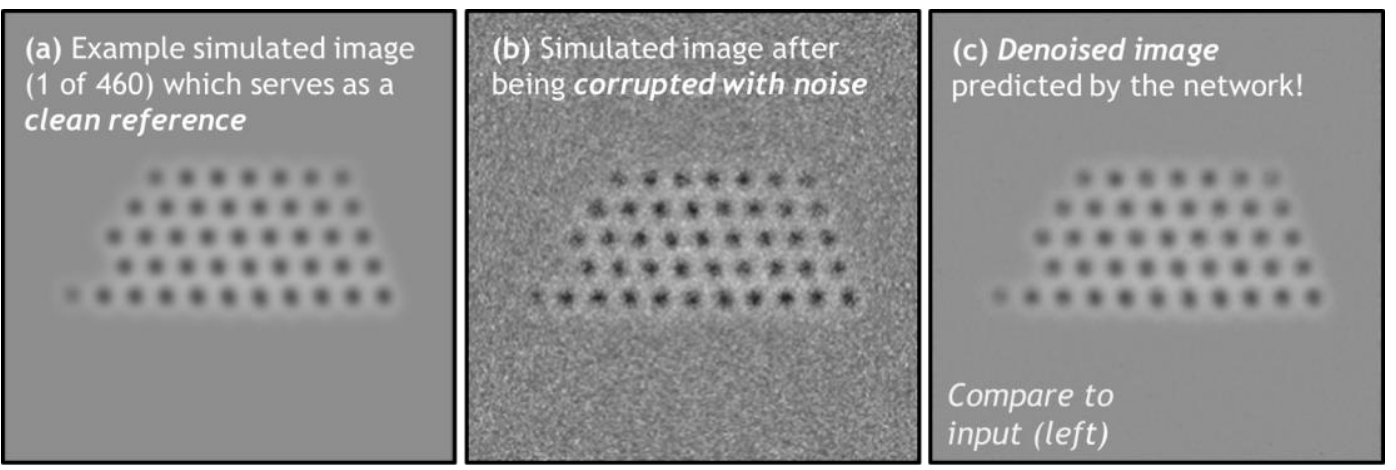

Figure 1. Training a convolutional neural network to denoise in situ TEM images. (a) Example simulated HRTEM image of a Pt nanoparticle which serves a clean reference or ground truth during training, (b) simulated image corrupted with a categorical noise model taken from the real images (see below), and (c) denoised image predicted by the trained network.

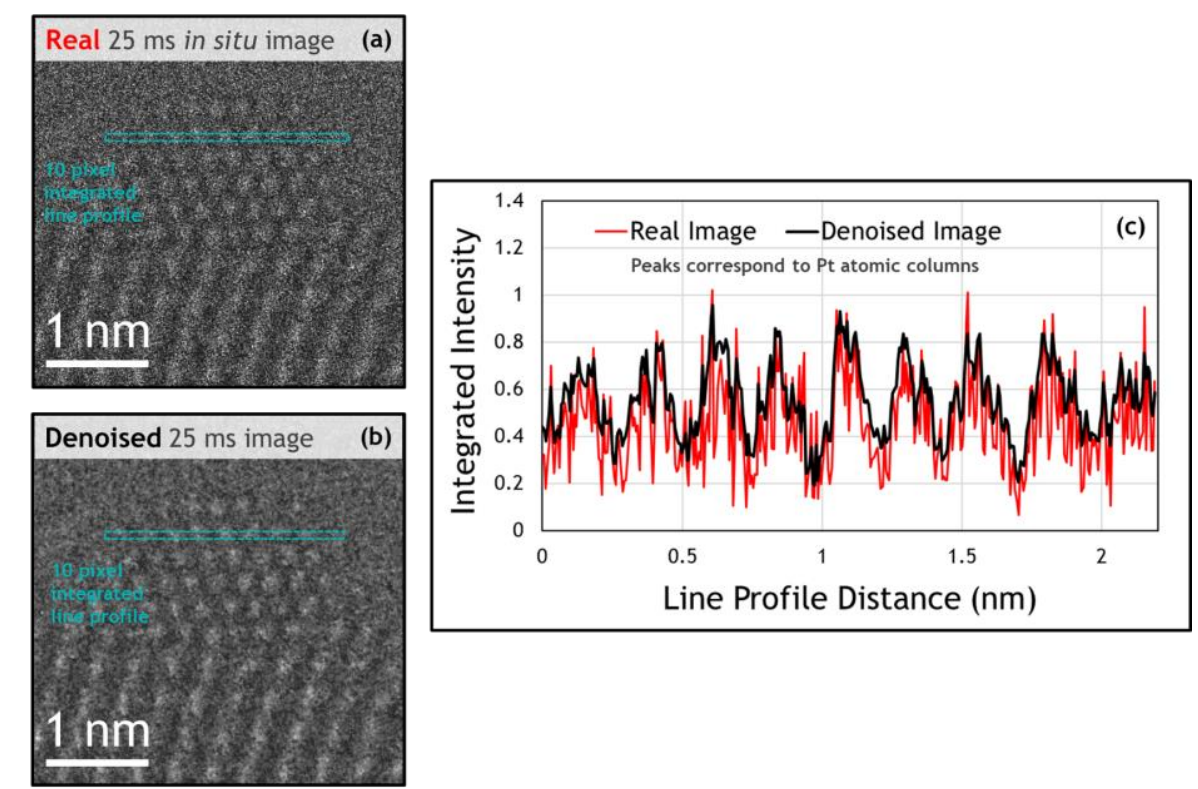

Figure 2. Comparing real and denoised in situ TEM images. (a) $25 \mathrm{~ms}$ image of a CeO2-supported Pt nanoparticle in 0.5 mTorr $\mathrm{N}_{2}$ gas, (b) denoised image which has been processed with the trained neural network, and (c) comparison of 10 pixel integrated line profiles calculated across identical regions in both images.

\section{References}

1. Somorjai, G. A., Langmuir, 1991. 7(12): p. 3176-3182.

2. Crozier, P. A., et al., Microscopy and Microanalysis, 2019. 25(S2): p. 1464-1465.

3. Faruqi, A. and G. McMullan, Nucl. Instrum. Methods Phys. Res, 2018. 878: p. 180-190.

4. J. Barthel, Ultramicroscopy, 2018. 193, p. 1-11.

5. S. Mohan, Z. Kadkhodaie, E. P. Simocelli, and C. Fernandez-Granda, 2019. Robust and Interpretable Blind Image Denoising via Bias-Free Convolutional Neural Networks, preprint available at https://arxiv.org/abs/1906.05478

6. We gratefully acknowledge support of NSF grants CBET-1604971 and OAC-1940263, and the facilities at ASU's John M. Cowley Center for High Resolution Electron Microscopy. 